\title{
Thin film like terahertz bolometric detector on Bi2212 single crystal
}

\author{
T. Semerci ${ }^{1} \cdot$ Y. Demirhan ${ }^{2} \cdot$ N. Miyakawa ${ }^{3} \cdot$ \\ H. B. Wang ${ }^{4} \cdot$ L. Ozyuzer ${ }^{2}$
}

Received: 9 October 2015/Accepted: 25 May 2016/Published online: 2 June 2016

(C) Springer Science+Business Media New York 2016

\begin{abstract}
In this study, we developed a microbolometer chip fabricated from high temperature superconducting $\mathrm{Bi}_{2} \mathrm{Sr}_{2} \mathrm{CaCu}_{2} \mathrm{O}_{8+\delta}$ ( $\mathrm{Bi2212}$ ) single crystals for the terahertz (THz) detection. For the manufacturing of the microbolometer chips, Bi2212 single crystals were transferred on substrate in the thin film like form and electron beam lithography, ion beam etching techniques were used. Resistance versus temperature behavior of the bolometer chips were performed by four probe technique in liquid nitrogen cryostat. Bi2212 microchips were integrated and characterized using in our custom-designed cryogenic bolometer system instead of expensive and massive cooling systems. The fabricated microchips significantly detected signals from the Stefan-Boltzmann lamp which includes a portion of $\mathrm{THz}$ radiation. The detected power and response time were studied for Bi2212 thin film like microbolometer chips. Our results demonstrated the feasibility of improved Bi2212 microchips could be used for bolometric detection for $\mathrm{THz}$ applications.
\end{abstract}

Keywords Terahertz (THz) · Bolometer · Response time · Bi2212 single crystal · High temperature superconductors

This article is part of the Topical Collection on TERA-MIR: Materials, Generation, Detection and Applications.

Guest Edited by Mauro F. Pereira, Anna Wojcik-Jedlinska, Renata Butkute, Trevor Benson, Marian Marciniak and Filip Todorov.

T. Semerci

tugcesemerci@iyte.edu.tr

1 Department of Materials Science and Engineering, Izmir Institute of Technology, 35430 Urla, Izmir, Turkey

2 Department of Physics, Izmir Institute of Technology, 35430 Urla, Izmir, Turkey

3 Department of Applied Physics, Tokyo University of Science, Tokyo, Japan

4 National Institute for Materials Science, Tsukuba 3050047, Japan 


\section{Introduction}

Terahertz $(\mathrm{THz})$ technology $(0.1-10 \mathrm{THz})$ is an extremely attractive research field with wide-range application areas such as security, medicine, quality control and environmental monitoring, but there is still lack of compact solid state sources, detectors and components (Tonouchi 2007; Ozyuzer et al. 2007; Kadowaki et al. 2010). In more recent times, progress in the development of $\mathrm{THz}$ sources in our group (Demirhan et al. 2015; Turkoglu et al. 2013, 2012) and pioneering efforts in the production of $\mathrm{THz}$ detectors have been observed. For detecting weak signals in the submillimeter or in some other words terahertz range, there exists a promising contestant namely superconducting Hot Electron Bolometers (HEBs). It is comprised of two thick metal pads that are connected by a small superconducting microbridge. By keeping HEBs in the transition state from superconductor to normal metal, a small change in temperature or absorbed power is attained and consequently a large change in resistance is observed which is a result of the creation of "hot electrons" on the bridge. The time $\mathrm{T}_{\text {th }}$ which is the time for the hot electrons to diffuse out of the bridge is called the characteristic reset time of the device and the thermal bandwidth of the device namely a cutoff frequency, $\gamma_{c}=\left(2 \pi T_{t h}\right)^{-1}$ is determined by this reset time. Responsivity, response time and easiness of operation are the most significant properties of a system which is based on HEB in addition to the above mentioned specifications. Moreover, from the point of view of obtaining best results for detectivity and sensitivity from the bolometer, the prominent parameters are the small heat capacity and large thermal conductance.

The relationship between heat capacity $(\mathrm{C})$ and thermal conductance $(\mathrm{G})$ is given by this formula:

$$
\text { Response time }(\mathrm{T})=\frac{\mathrm{C}}{\mathrm{G}}
$$

It is a well-known fact that superconducting bolometers have smaller response time compared to other detectors. Various approaches have been initiated to develop HEB from superconducting materials.

For the production of bolometric detectors which have smaller response times, $\mathrm{Nb}$ and $\mathrm{NbN}$ superconducting thin film is mostly preferred. At $190 \mathrm{mK}$ a time constant of $25 \mu \mathrm{s}$ has been given for $\mathrm{Nb}$ bolometer. It has been demonstrated that, a bolometer produced from $\mathrm{NbN}$ superconducting film has a smaller response time than $\mathrm{Nb}$ films (Karasik et al. 1999). Later on, in the year 2000, ultrathin $3 \mathrm{~nm} \mathrm{NbN}$ film bolometer with 30 ps response time was achieved (Ilin et al. 2000). By using high temperature superconductor $\mathrm{YBa}_{2}$ $\mathrm{Cu}_{3} \mathrm{O}_{7-\mathrm{x}}$ (YBCO) thin film for the production of the bolometer, Hammar et al. acquired response times almost at the range of picoseconds. Moreover, they demonstrated that responsivity is decreased when increasing the antenna structure which $1.5 \mu \mathrm{m} \times 1.5 \mu \mathrm{m}$ area has nearly $180 \mathrm{~V} / \mathrm{W}$ and $4 \mu \mathrm{m} \times 1 \mu \mathrm{m}$ area has $100 \mathrm{~V} / \mathrm{W}$ as responsivity (Hammar et al. 2011). MgB HEBs operating at THz frequency which are produced from $20 \mathrm{~nm}$ films on silicon substrates has been achieved as well (Cherednichenko et al. 2007).

$\mathrm{Bi}_{2} \mathrm{Sr}_{2} \mathrm{CaCu}_{2} \mathrm{O}_{8+\delta}(\mathrm{Bi} 2212)$ is a high temperature superconductor with critical temperature around $95 \mathrm{~K}$. This critical temperature value is changed with oxygen doping level. Bi2212 single crystal has layered structure property and it is possible to obtain large crystal. It is easy to get large single crystals on the other hand it is hard to grow Bi2212 thin film without any defect or impurities due to large anisotropic properties of the material. 
Because of this difficulty, we transferred Bi2212 single crystal on sapphire substrate as a thin film.

To the best of our knowledge, in the literature; there is no HEB from high- $\mathrm{T}_{\mathrm{c}}$ superconductor Bi2212 single crystals. In this study, a micron sized chip of the bolometric detector was fabricated and developed from Bi2212 single crystals. The bolometric response of our detector on $\mathrm{THz}$ waves have been investigated.

\section{Experimental details}

In the fabrication part of this study, a piece of Bi2212 single crystal was glued onto a sapphire substrate from its smooth a-b surface by two component fast dry epoxy. In the literature, many different adhesive materials were used to transfer superconducting crystals on substrate such as silver epoxy (Turkoglu et al. 2012; Koseoglu et al. 2011), polyimide and non-photosensitive polyimide PIX (Yamada et al. 2007; You et al. 2005; Wang et al. 2001; Yamaki et al. 2011). The preference of adhesive can affects response time of bolometer, and the chosen epoxy has to be compatible with temperature cycling. In order to get a fresh and smooth surface on Bi2212, the crystal was cleaved layer by layer with a scotch tape. This cleaved process is occurred from the weakest layer which is $\mathrm{BiO}$ layer in Bi2212 single crystal (Yamaki et al. 2011). Transferred Bi2212 thin film like crystals were kept in air until dry and cut their edges as pyramids form to coat Bi2212 single layer with gold. The crystals were shadowed with aluminum foil for providing four point contact area to get rid of contact resistance (Fig. 1). Au layer with the thickness of 150-200 nm was thermally evaporated on the cleaved crystal surface immediately to prevent the chemical reactions (Kakeya et al. 2008).

For further processing, micro bridges on Bi2212 thin film like crystals have been fabricated using clean room facilities, conventional e-beam lithography and argon ion beam etching techniques. The ambient black part indicates sapphire substrate while the middle bright part is the Bi2212 thin film like crystal with $100 \mu \mathrm{m}$ micro bridge as shown in Fig. 2. Micro bridge structure has a smooth surface which has no defects, impurities, cracks, etc. Moreover, 4 point contact with silver epoxy by gold wires does not cause any contact resistance.

We have composed a custom-designed, compact and nonexpensive cryostat system for both electrical and bolometric measurements. The system consists of a cylindrical outer chamber which has $250 \mathrm{~mm}$ height and $200 \mathrm{~mm}$ diameter and a liquid nitrogen chamber in the inner part; $153 \mathrm{~mm}$ diameter, $150 \mathrm{~mm}$ height with $3 \mathrm{~L}$ capacity to cool our chip by

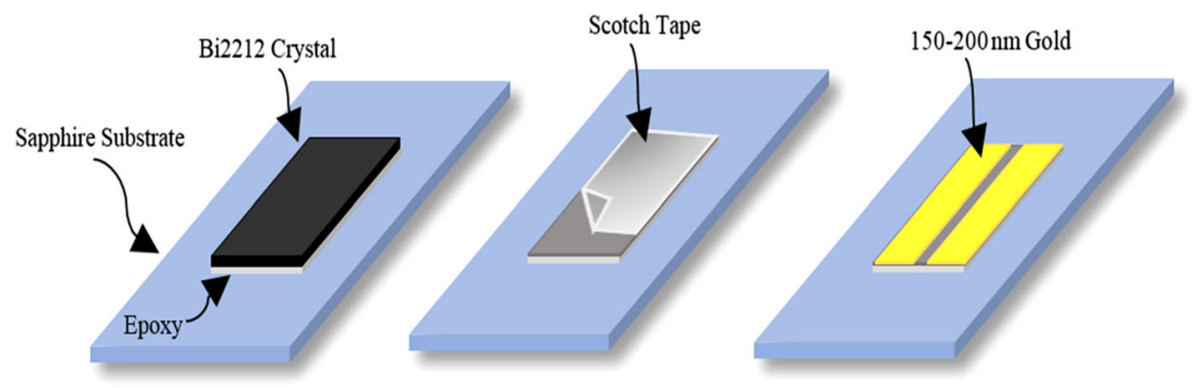

Fig. 1 Steps of fabrication for Bi2212 microchips with transferring, cleaving and deposition processes 


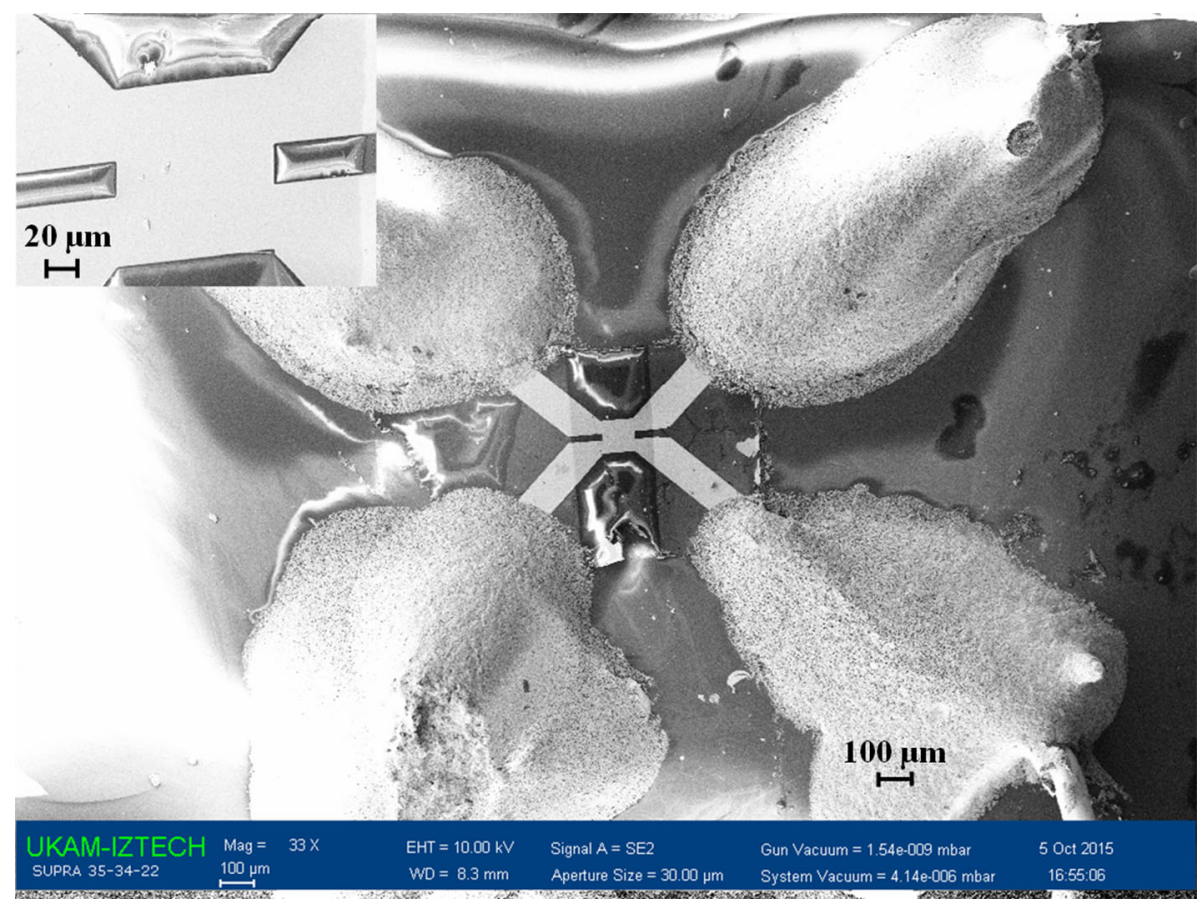

Fig. 2 SEM image one of the fabricated microchip with Bi2212 thin film like crystal on sapphire substrate. The inset shows to zoom on the microbridge

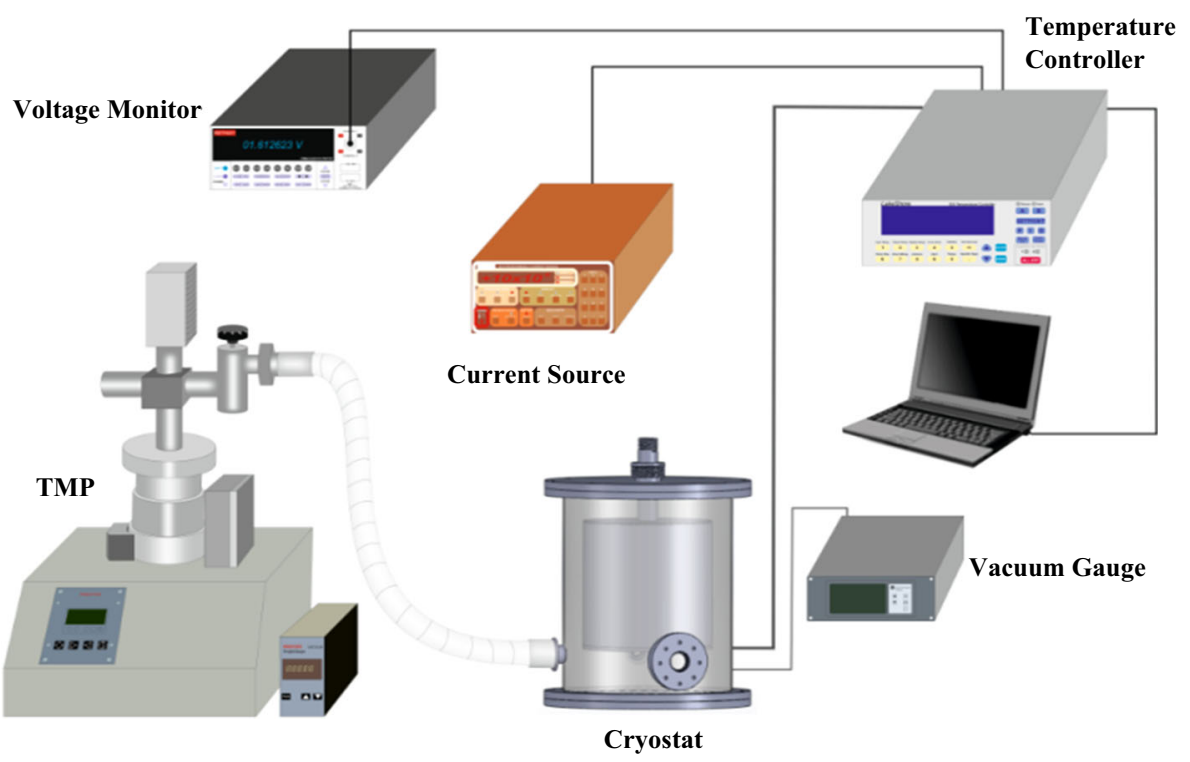

Fig. 3 Schematic view of electrical measurement setup 
liquid nitrogen. Fabricated microchips were placed to the holder which is located in the liquid nitrogen chamber and has to be stored in nitrogen environment for long durations during the measurements. Liquid nitrogen chamber was surrounded with $15 \mu \mathrm{m}$ metalize film 4 times for thermal insulation purpose and by this way we can hold liquid nitrogen inside the chamber without vaporization up to $30 \mathrm{~h}$. Electrical measurement setup which is shown in Fig. 3 was used to determine the critical temperature and superconducting transition region of chips before bolometric measurements.

It is important to observe the sharp resistance decrease near the critical temperature of superconducting crystals to begin to the bolometric measurements. In this region, middle point of the superconducting transition was selected approximately as operating temperature. Bolometric measurement set up which is shown in Fig. 4 was used for characterization and to determine the response times of chips.

When temperature of chip was setting to the operating temperature by heater, at this operating temperature, Stefan Boltzmann Lamp sent a pulsed radiation at certain times. These electromagnetic waves was passed through the Polyethylene (PE) window and reached to the integrated microchip.

We have used a Bruker Vertex 80v Fourier Transform Infrared Spectrometer (FTIR) with DTGS detector using Mylar beam splitters for attenuation measurement of the PE window in this study. Measurement chamber was evacuated below $3 \mathrm{hPa}$ during both background and sample measurements, resolution were $2 \mathrm{~cm}^{-1}$. FTIR measurement of PE window was shown in Fig. 5 that average transmittance of the PE window is equal to about $80 \%$ in the frequency range $0.2-1.6 \mathrm{THz}$. After electrical and bolometric measurement were done by using set-up in Fig. 3, signals were focused onto the chip by wave collector as shown in Fig. 4 and a little variation in resistance values were observed during sending signal from Stefan-Boltzmann Lamp. These variation of resistance were observed via Labview program and occurred because of focused signal increase the temperature of the

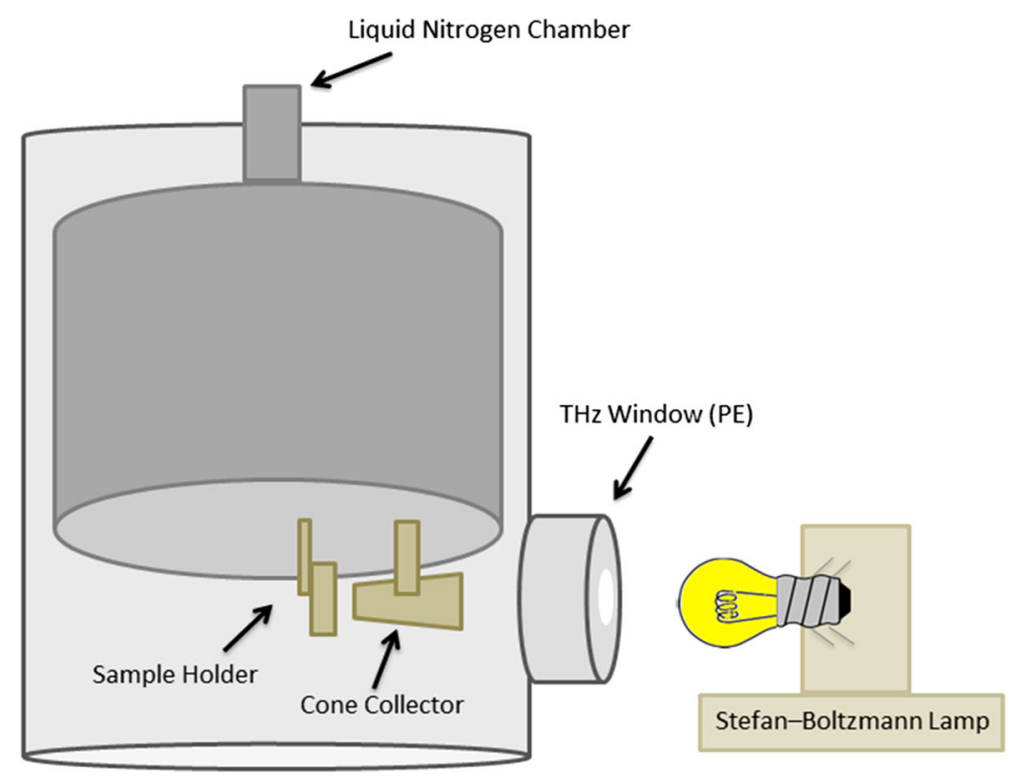

Fig. 4 Schematic view of bolometric measurement setup 
Fig. 5 FTIR measurement of Polyethylene window used in cryostat system

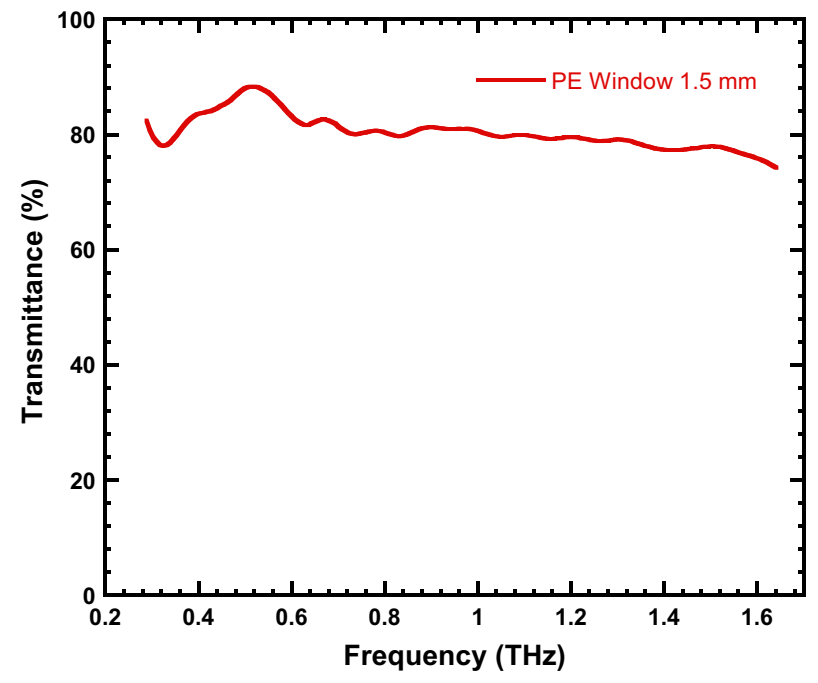

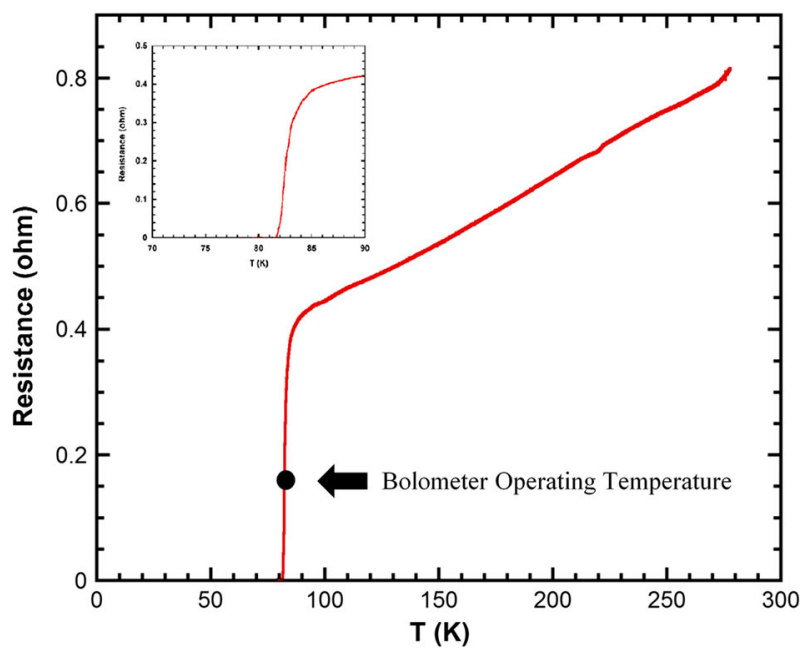

Fig. 6 Resistance versus temperature behavior of a Bi2212 thin film like crystal chip. The black dot corresponds to the operating temperature value of this particular microchip bolometer. The inset shows to zoom in the superconducting transition region

microbridge region that cause the increase of resistance. After bolometric measurements, response time was calculated from resistance-time graphs.

\section{Results and discussion}

Resistance versus temperature $(\mathrm{R}-\mathrm{T})$ characteristics along the a-b axis of the microchips was measured in our designed cryostat system. During the measurement constant $10 \mu \mathrm{A}$ current is applied along to samples. In our R-T measurements, we have usually observed a 
sharp superconducting transition around $90 \mathrm{~K}$. The graph in Fig. 6 exhibit a typical temperature dependence of the a-b axis resistance of Bi2212 thin film like crystal chip, which tends to decrease with decreasing temperature around room temperature and turns to sharp increase near critical temperature. It is important to carry out the bolometric measurements with Bi2212 thin film like crystal chips which have sharp superconducting transition.

Electrical measurements also show that the resistance values depend on the temperature, which can be used to determine onset and zero of resistance, critical temperature, etc. These critical parameters for electrical and bolometric measurements are shown in Table 1. Each sample has different onset temperature depending on oxygen doping level of the Bi2212 crystals. Our Bi2212 thin film like crystals' thickness values were between 100 and $500 \mathrm{~nm}$. During the cleavage process, we are not able to adjust the thickness of the thin film like crystals in a well-controlled way. It has been observed that the onset and zero of resistance values of the electrical measurements depend on the thickness of the Bi2212 crystals (You et al. 2006). The chip resistance was hold at certain temperature value via heater at the operating temperature during the bolometric measurements. Operating temperature was selected according to the superconducting transition region of each sample, it was set to the temperature value in the middle point of the superconducting transition region. Each sample has their own operating resistance values with respect to their superconducting transition curves which was equal to the initial resistance of the bolometric measurements. Bolometric measurements were performed by switching on-off a Stefan-Boltzmann Lamp using a square wave shape power.

Figure 7 represents the bolometric measurement of sample Bol-a, chip's critical temperature, operating temperature and resistance values were as $90 \mathrm{~K}, 84 \mathrm{~K}$ and $0.552 \Omega$ given respectively in Table 1. During the measurement, Stefan-Boltzmann Lamp was switched on and off once every $40 \mathrm{~s}$ and $8 \mathrm{~m} \Omega$ resistance change was observed for sample Bol-a. Response time was calculated for each sample by defining the initial resistance point as reference point which was almost zero. Response time is the elapse time 1/e of the moment of switch off the signal. Calculated response time of this chip was $825 \mathrm{~ms}$ which is shown in Fig. 7. Calculated response time values of other fabricated chips are presented in Table 1, as $1.910,1.150,0.825$ and $0.972 \mathrm{~s}$ depending on the superconducting critical parameters (onset and zero of resistance, critical temperature of chip). The calculated response time of the microchips were consistently higher than the expected value related to the limitation of the data transfer speed in our measurement set up, lock-in-amplifier might be used to provide more accurate results.

To improve the sensitivity of bolometric detection, antenna structures can be integrated to microchips designed for the THz range (Cibella et al. 2009). Besides, acquired response time can be improved by reducing the dimensions of microbridge as the working principle

Table 1 Electrical properties and response times of our selected samples

\begin{tabular}{llllll}
\hline & $\begin{array}{l}\mathrm{R}_{300 \mathrm{~K}} \\
(\mathrm{ohm})\end{array}$ & $\begin{array}{l}\mathrm{R}_{\mathrm{Tc}} \text { (onset) } \\
(\mathrm{ohm})\end{array}$ & $\begin{array}{l}\mathrm{T}_{\mathrm{c}} \text { (onset) } \\
(\mathrm{K})\end{array}$ & $\Delta \mathrm{T}_{\mathrm{c}}(\mathrm{K})$ & $\begin{array}{l}\text { Response } \\
\text { time }(\mathrm{s})\end{array}$ \\
\hline Bol-a & 1.60 & 1.10 & 90 & 6.11 & 1.910 \\
Bol-b & 0.81 & 0.44 & 87 & 4.20 & 1.150 \\
Bol-c & 5.40 & 2.10 & 90 & 8.32 & 0.825 \\
Bol-d & 0.72 & 0.31 & 86 & 4.10 & 0.972 \\
\hline
\end{tabular}


Fig. 7 Change of resistance under the influence of signal

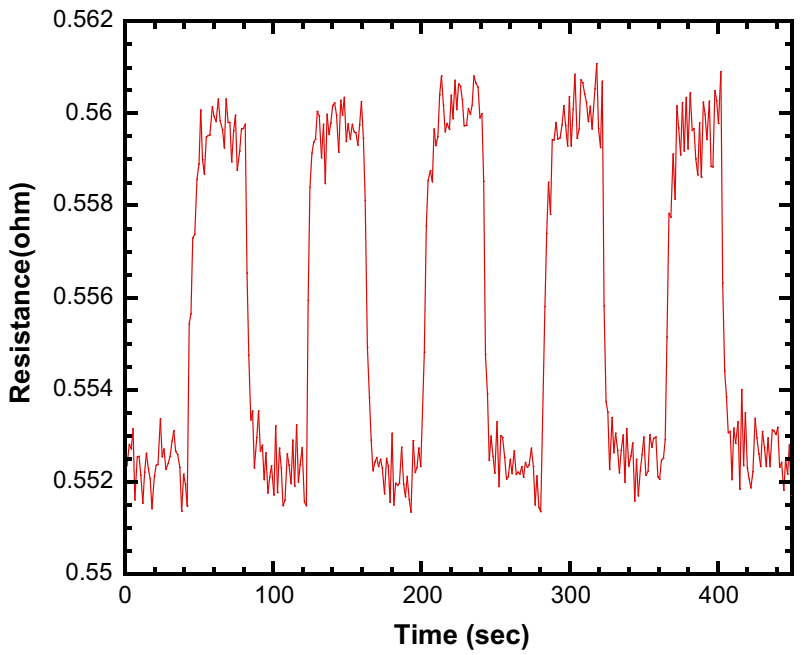

Fig. 8 Change of the microbridge resistance with different signal power

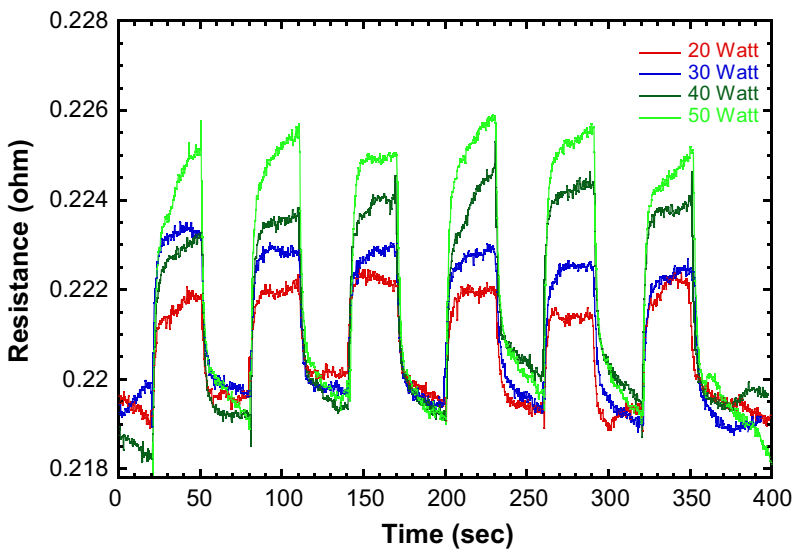

of the detectors based on electron-phonon interaction time. It has been observed that length of device and bridge affect the response time of bolometers. (Burke et al. 1996). The length of the microbridge must be as short as possible, which is related to maximum value $L_{\max }=2\left(D_{e} T_{e e}\right)^{1 / 2}$, where $D_{e}$ is the diffusion constant, $T_{e e}$ is the interaction time (Burke et al. 1996; Bozbey et al. 2006). Response time of diffusion-cooled type bolometers has been presented by Prober (1993). YBCO high temperature superconducting thin film based microbolometer for $\mathrm{THz}$ radiation was demonstrated and response time of 300 ps was achieved (Hammar et al. 2011). In addition to these, antenna structures were fabricated to provide higher sensitivity from the detectors (Mendis et al. 2004; Hosseini et al. 2011). In recent studies, bolometric detection from YBCO grain boundary Josephson junction has been realized where Bi2212 intrinsic Josephson junction was used as emitter (An et al. 2013). In our study, it is proved that Bi2212 single crystals can be used for detection purpose. 
In order to investigate the microbridge response to incoming optical power, we have performed bolometric measurements by adjusting power to 20, 30, 40, 50 Watt respectively during constant time period, $30 \mathrm{~s}$. When properly oriented, a linear relationship was observed between the Stephan Boltzmann lamp intensity and final response resistance values of the microchips during measurements as shown in Fig. 8. It was proved that the final response resistance of the microchips is increased via increasing power of the signal from the source. As expected, initial resistance of the microchips did not change by increasing intensity of the signals. Note that these power values do not correspond to $\mathrm{THz}$ emission power. Stefan-Boltzmann Lamp creates a wide spectrum of electromagnetic waves and only small portion of spectrum corresponds to $\mathrm{THz}$ waves which is much smaller than these power values.

After carrying out the bolometric measurements with increasing power level, it should be determined that which power value gives the nearly zero resistance. When we curve fit our "change of resistance with different signal power" graph, we found that the power of 1.95 Watt refers to zero resistance as shown in Fig. 9. It means that our bolometric detector has sensitivity until the 1.95 Watt power. Above this power level, detector can show only little resistance change in return signals which is negligible and microchips are no longer available for bolometric detection.

\section{Conclusion}

In summary, we have fabricated microchips from Bi2212 single crystals for bolometric detection. While 100-500 $\mathrm{nm}$ thick crystals are readily obtainable and can be transferred to the substrates, we have fabricated micro bridges from thin film like Bi2212 single crystals instead of Bi2212 thin films. Besides, it is hard to fabricate high-quality Bi2212 thin films with strong superconducting properties and good crystallinity as single crystals. We have carried out electrical and bolometric measurements in a custom-designed, nitrogen cooled cryostat system. The calculated response times of the chips were between 1.910 and $0.825 \mathrm{~s}$, consistently higher than the expected value. We estimated that this might be directly correlated with the limitation of the data transfer speed in our set up, lock-inamplifier might be used to provide more accurate results. Briefly, we have demonstrated the ability of bolometric detection from Bi2212 single crystals and our experimental results

Fig. 9 Curve fit graph of resistance-power measurement

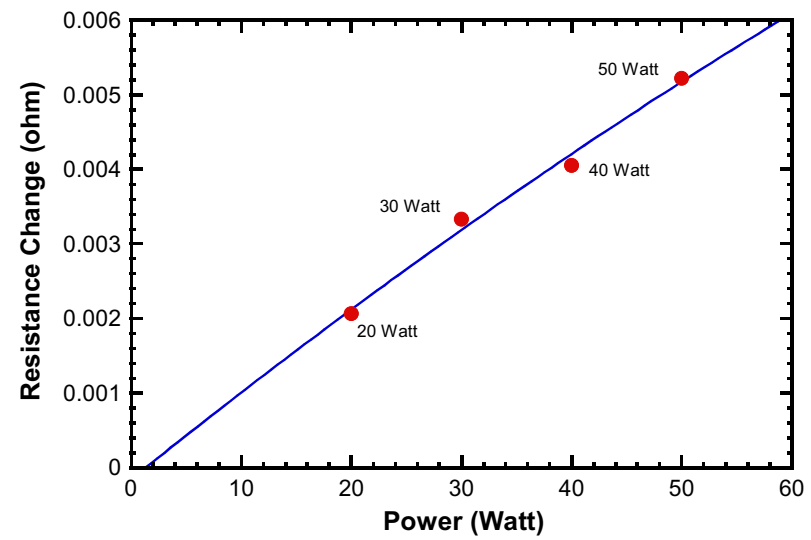


reveal that Bi2212 single crystals are good candidates for bolometric detection purpose. The detection properties of micro bolometer chips can be further improved by fabricating a nano-sized bridge in the middle part of the chip, integrating an antenna and filters structures.

Acknowledgments This research was partially supported by Ministry of Science, Industry and Technology under project number SANTEZ 1386.STZ.2012-1 which was cooperated with ASELSAN. We also thank the Applied Quantum Research Center of IZTECH for the research facilities they offered.

\section{References}

An, D.Y., Yuani, J., Kinev, N., Li, M.Y., Huang, Y., Ji, M., Zhang, H., Sun, Z.L., Kang, L., Jin, B.B., Chen, J., Li, J., Gross, B., Ishii, A., Hirata, K., Hatano, T., Koshelets, V.P., Koelle, D., Kleiner, R., Wang, H.B., Xu, W.W., Wu, P.H.: Terahertz emission and detection both based on high-Tc superconductors: towards an integrated receiver. Appl. Phys. Lett. 102, 092601 (2013)

Bozbey, A., Fardmanesh, M., Schubert, J., Banzet, M.: Superconductivity transition dependence of the thermal crosstalk in $\mathrm{YBa}_{2} \mathrm{Cu}_{3} \mathrm{O}_{7 \mathrm{x}}$ edge-transition bolometer arrays. IEEE Trans. Appl. Supercond. 16(1), 9-14 (2006)

Burke, P.J., Schoelkopf, R.J., Prober, D.E., Skalare, A., McGrath, W.R., Bumble, B., Le Duc, H.G.: Length scaling of bandwidth and noise in hot-electron superconducting mixers. Appl. Phys. Lett. 68(23), 3344-3346 (1996)

Cherednichenko, S., Drakinskiy, V., Ueda, K., Naito, M.: Terahertz mixing in MgB microbolometers. Appl. Phys. Lett. 90, 023507 (2007)

Cibella, S., Carelli, P., Castellano, M.G., Foglietti, V., Leoni, R., Ortolani, M., Torrioli, G.A.: Superconducting bolometer antenna-coupled to terahertz waves. J. Low Temp. Phys. 154(5-6), 142-149 (2009)

Demirhan, Y., Saglam, H., Turkoglu, F., Alaboz, H., Ozyuzer, L., Miyakawa, N., Kadowaki, K.: Area dependence and influence of crystal inhomogeneity on superconducting properties of Bi2212 mesa structures. Vacuum 120(Part B), 89-94 (2015)

Hammar, A., Cherednichenko, S., Bevilacqua, S., Drakinskiy, V., Stake, J.: Terahertz direct detection in $\mathrm{YBa}_{2} \mathrm{Cu}_{3} \mathrm{O}_{7}$ microbolometers. IEEE Trans. Terahertz Sci. Technol. 1(2), 390-394 (2011)

Hosseini, M., Moftakharzadeh, A., Kokabi, A., Vesaghi, M.A., Kinder, H., Fardmanesh, M.: Characterization of a transition-edge bolometer made of YBCO thin films prepared by nonfluorine metal-organic deposition. IEEE Trans. Appl. Supercond. 21(6), 3587-3591 (2011)

Ilin, K.S., Lindgren, M., Currie, M., Semenov, A.D., Goltsman, G.N., Sobolewski, R., Cherednichenko, S.I., Gershenzon, E.M.: Picosecond hot-electron energy relaxation in $\mathrm{NbN}$ superconducting photodetectors. Appl. Phys. Lett. 76, 2752-2754 (2000)

Kadowaki, K., Tsujimoto, M., Yamaki, K., Yamamoto, T., Kashiwagi, T., Minami, H., Tachiki, M., Klemm, R.A.: Evidence for a dual-source mechanism of terahertz radiation from rectangular mesas of single crystalline $\mathrm{Bi}_{2} \mathrm{Sr}_{2} \mathrm{CaCu}_{2} \mathrm{O}_{8+\delta}$ intrinsic Josephson junctions. J. Phys. Soc. Jpn. 79, 023703 (2010)

Kakeya, I., Fukui, K., Kawamata, K., Yamamoto, T., Kadowaki, K.: Quantum oscillation of the c-axis resistivity due to entrance of pancake vortices into micro-fabricated $\mathrm{Bi}_{2} \mathrm{Sr}_{2} \mathrm{CaCu}_{2} \mathrm{O}_{8+\mathrm{d}}$ intrinsic Josephson junctions. Phys. C 468, 669-673 (2008)

Karasik, B.S., McGrath, W.R., Wyss, R.A.: Optimal choice of materials for HEB superconducting mixers. IEEE Transc. Appl. Supercond. 9, 4213-4216 (1999)

Koseoglu, H., Turkoglu, F., Simsek, Y., Ozyuzer, L.: The fabrication of THz emitting mesas by reactive ionbeam etching of superconducting Bi2212 with multilayer masks. J. Supercond. Nov. Magn. 24(1-2), 1083-1086 (2011)

Mendis, R., Sydlo, C., Sigmund, J., Feiginov, M., Meissner, P., Hartnagel, H.L.: Tunable CW-THz system with a log-periodic photoconductive emitter. Solid-State Electron. 48(10-11), 2041-2045 (2004)

Ozyuzer, L., Koshelev, A.E., Kurter, C., Gopalsami, N., Li, Q., Tachiki, M., Kadowaki, K., Tamamoto, T., Minami, H., Yamaguchi, H., Tachiki, T., Gray, K.E., Kwok, W.K., Welp, U.: Emission of coherent $\mathrm{THz}$ radiation from superconductors. Science 318, 1291-1293 (2007)

Prober, D.E.: Superconducting terahertz mixer using a transition-edge microbolometer. Appl. Phys. Lett. 62(17), 2119-2121 (1993)

Tonouchi, M.: Cutting-edge terahertz technology. Nat. Photonics 1, 97-105 (2007) 
Turkoglu, F., Koseoglu, H., Demirhan, Y., Ozyuzer, L., Preu, S., Malzer, S., Simsek, Y., Muller, P., Yamamoto, T., Kadowaki, K.: Interferometer measurements of terahertz waves from $\mathrm{Bi}_{2} \mathrm{Sr}_{2} \mathrm{CaCu}_{2-}$ $\mathrm{O}_{8+\mathrm{d}}$ mesas. Supercond. Sci. Technol. 25, 125004 (2012)

Turkoglu, F., Ozyuzer, L., Koseoglu, H., Demirhan, Y., Preu, S., Malzer, S., Simsek, Y., Wang, H.B., Muller, P.: Emission of the THz waves from large area mesas of superconducting $\mathrm{Bi}_{2} \mathrm{Sr}_{2} \mathrm{CaCu}_{2} \mathrm{O}_{8+\mathrm{d}}$ by the injection of spin polarized current. Phys. C 491, 7-10 (2013)

Wang, H.B., Wu, P.H., Yamashita, T.: Stacks of intrinsic Josephson junctions singled out from inside $\mathrm{Bi}_{2} \mathrm{Sr}_{2} \mathrm{CaCu}_{2} \mathrm{O}_{8+\mathrm{x}}$ single crystals. Appl. Phys. Lett. 78(25), 4010-4012 (2001)

Yamada, Y., Watanabe, T., Suzuki, M.: Fabrication and transport properties for cleaved thin film BSCCO single crystals. IEEE Trans. Appl. Supercond. 17(2), 3533-3536 (2007)

Yamaki, K., Tsujimoto, M., Yamamoto, T., Furukawa, A., Kashiwagi, T., Minami, H., Kadowaki, K.: Highpower terahertz electromagnetic wave emission from high-T-c superconducting $\mathrm{Bi}_{2} \mathrm{Sr}_{2} \mathrm{CaCu}_{2} \mathrm{O}_{8}+\mathrm{d}$ mesa structures. Opt. Express 19(4), 3193-3201 (2011)

You, L.X., Yurgens, A., Winkler, D., Lin, C.T., Liang, B.: Superconducting properties of ultrathin Bi ${ }_{2-}$ $\mathrm{Sr}_{2} \mathrm{CaCu}_{2} \mathrm{O}_{8+\mathrm{x}}$ single crystals. J. Appl. Phys. 98, 033913 (2005)

You, L.X., Yurgens, A., Winkler, D., Lin, C.T., Liang, B.: Thickness dependence of the superconducting properties of ultra-thin $\mathrm{Bi}_{2} \mathrm{Sr}_{2} \mathrm{CaCu}_{2} \mathrm{O}_{8+\mathrm{x}}$ single crystals. Supercond. Sci. Technol. 19(5), S205-S208 (2006) 